\title{
Normally Hearing Subjects Have No Advantage of Better Audiograms in Listening Tasks
}

\author{
P. KleczKowski* and M. Pluta \\ AGH University of Science and Technology, Faculty of Mechanical Engineering and Robotics \\ Department of Mechanics and Vibroacoustics, al. A. Mickiewicza 30, 30-059 Krakow, Poland
}

\begin{abstract}
Some correlation has been found in literature between the amount of mild hearing impairment and lowering of performance in listening tasks. The relationship between measures of hearing acuity and performance in listening tasks in the population of normally hearing subjects has not found a solid evidence. In this work six one-parameter measures of hearing acuity based on audiograms, including three originally proposed by the authors, were used to investigate whether a relationship between those measures and listeners' performance existed. The results of six listening tests were investigated, three with speech, two with musical excerpts and one with everyday sounds. The results showed no correlation between hearing acuity and performance, with the exception of everyday sounds, where some correlation was found. No significant differences between the measures used were found.
\end{abstract}

PACS: $43.66 .-\mathrm{x}$

\section{Introduction}

Several one-parameter measures derived from the results of standard audiometry have been in use. Most of them are suited to evaluate the ability to comprehend speech. They are usually derived as an average of positive threshold values in middle frequency bands, crucial for speech. Often used methods employ three or four bands in the better ear, while the Fletcher method [1] used only two bands out of $0.5,1$, and $2 \mathrm{kHz}$, and took the average of the two smallest values of loss. Fletcher argued that his method was superior, especially for "non-flat" audiograms. The "AMA" (American Medical Association) formula gives the percentage of hearing loss. It has undergone several modifications and is suitable for impairments greater than $25 \mathrm{~dB}$ on the average in frequency bands $0.5,1,2$, and $3 \mathrm{kHz}$ [2].

A relation between the results of pure tone and speech reception tests has been of interest of audiologists [3, 4]. Carhart [3] found a correlation of 0.59 between the AMA formula (of 1947) and speech reception threshold (SRT) and a correlation of 0.69 between a method based on the average from three frequencies and the SRT. Siegenthaler and Strand [4] found even higher correlations between 0.7 and 0.8 . Both these analyses were carried out on patients treated at hospitals, with very mild to profound hearing impairments. The effect of hearing impairment on speech reception is easily accounted for. It is of interest however, whether there is any relationship between hearing acuity and the results of listening tests for listeners with normal hearing.

\footnotetext{
* corresponding author; e-mail: kleczkow@agh.edu.pl
}

It is usually accepted that only listeners with normal hearing should participate in listening tests [5]. A rule in psychoacoustic experiments with normally hearing subjects is that they should have audiometric thresholds better than $20 \mathrm{~dB}$ HL at octave frequencies from 250 through $8000 \mathrm{~Hz}$. Some research centres set a lower threshold: 15 or even $10 \mathrm{~dB}$. Some works have investigated the effect of hearing acuity on the results of listening tests in audio. In Refs. [6, 7] a two-parameter, audiogram-based measure of hearing acuity was used: the average for frequencies below $1 \mathrm{kHz}$ and for frequencies from $1 \mathrm{kHz}$ above. Listeners including those with mild hearing impairment rated the quality of loudspeakers [6]. Correlation $(r=0.65)$ between standard deviation of answers and listeners' hearing threshold was found below $1 \mathrm{kHz}$, but no correlation $(r=0.1)$ above $1 \mathrm{kHz}$. In a similar experiment, participated only by subjects fulfilling a $15 \mathrm{~dB}$ margin rule, that effect was not confirmed [7]. In some other works ([5] and references therein) no clear relationship between hearing acuity and the performance in the assessment of digital audio coding systems was found. In Ref. [8] the authors assumed that evaluation of subtle audio coding artifacts required particular acuity at frequencies over $4 \mathrm{kHz}$. They examined a group of listeners chosen as expert listeners with specific criteria and found that half of them had better but the other half had worse high frequency audiometric thresholds than the population's average.

In this work six one-parameter measures of hearing acuity based on audiograms were used. Three were originally proposed by the authors. The effect of the threshold of hearing on performance was investigated by analysing the results of six listening tasks. Three of them con- 
sisted in speech recognition and the other three consisted in recognition of subtle differences in audio. The panel of listeners included the percentage of those with formal mild hearing loss that is typical for the population of young listeners: $8 \%$, but their $20 \mathrm{~dB}$ thresholds were just exceeded.

\section{Method}

The following six one-parameter audiogram-based estimates of hearing acuity were used in this study (for all estimates, the value for the better ear was assumed).

(1) The average of audiometric thresholds in frequency bands: $0.5,1,2$, and $3 \mathrm{kHz}$, only for positive values (deficiencies in hearing). In cases of negative values 0 was assumed. This estimate has the same basis as the AMA formula. It was further abbreviated as: AAM4flo, for Audiogram-Average Mean 4 frequencies, losses only.

(2) The average of audiometric thresholds in frequency bands: $0.5,1,2$, and $3 \mathrm{kHz}$, including negative values, abbreviated as: AAM4f.

(3) The average of audiometric thresholds in frequency bands: $0.125,0.25,0.5,0.75,1,1.5,2,3,4,6$, and $8 \mathrm{kHz}$, including negative values. In this estimate, for each frequency either the threshold of the left or right ear was taken, whichever was lower. It was abbreviated as: AAMaf.

The next three estimates were designed by the authors in order to find out whether there is a relationship between "flatness" of the audiogram and the efficiency of hearing. The standard deviation of hearing thresholds was used as a natural measure of "flatness".

(4) Standard deviation of audiometric thresholds in frequency bands: $0.5,1,2$, and $3 \mathrm{kHz}$, only for positive values. In cases of negative values 0 was assumed. This was abbreviated as: AASD4flo.
(5) The average of audiometric thresholds in frequency bands: $0.5,1,2$ and $3 \mathrm{kHz}$, including negative values, abbreviated as: AASD4f.

(6) Standard deviation of audiometric thresholds in all frequency bands listed in (3), including negative values, abbreviated as: AASDaf.

Estimates (1), (2), (4) and (5) are adequate for speech signals, while estimates (3) and (6) are adequate for non-speech broadband signals. The list of estimates and abbreviations is given in Table $\mathrm{I}$.

Complete audiograms of 51 subjects, all of them students of Acoustic Engineering major at the AGH University of Science and Technology, were obtained in the same conditions.

TABLE I

The list of audiogram-based estimates of hearing acuity with abbreviations.

\begin{tabular}{|c|c|c|}
\hline \multicolumn{2}{|c|}{ Abbreviation } & Method of audiogram averaging \\
\hline 1 & AAM4flo & $\begin{array}{l}\text { Audiogram-Average Mean } 4 \text { fre- } \\
\text { quencies, losses only }\end{array}$ \\
\hline 2 & AAM4f & $\begin{array}{l}\text { Audiogram-Average Mean } 4 \text { fre- } \\
\text { quencies }\end{array}$ \\
\hline 3 & AAMaf & $\begin{array}{l}\text { Audiogram-Average Mean all fre- } \\
\text { quencies }\end{array}$ \\
\hline 4 & AASD4flo & $\begin{array}{l}\text { Audiogram-Average Standard De- } \\
\text { viation } 4 \text { frequencies, losses only }\end{array}$ \\
\hline 5 & AASD4f & $\begin{array}{l}\text { Audiogram-Average Standard De- } \\
\text { viation } 4 \text { frequencies }\end{array}$ \\
\hline 6 & AASDaf & $\begin{array}{l}\text { Audiogram-Average Standard De- } \\
\text { viation all frequencies }\end{array}$ \\
\hline
\end{tabular}

TABLE II

Correlation coefficients for the relationship of performance in the listening task vs. estimate of hearing acuity. Asterisks denote statistically insignificant correlations.

\begin{tabular}{l|c|c|c|c|c|c}
\hline \hline \multirow{2}{*}{$\begin{array}{c}\text { Type of stimulus / } \\
\text { Number of participants }\end{array}$} & \multicolumn{7}{|c}{ Type of audiogram average } \\
\cline { 2 - 7 } & AASDaf & AAMaf & AASD4f & AAM4f & AASD4flo & AAM4flo \\
\hline Individual words / 22 & $0.249^{*}$ & $-0.211^{*}$ & $-0.015^{*}$ & $0.063^{*}$ & $0.087^{*}$ & $0.203^{*}$ \\
Triplets / 19 & $0.191^{*}$ & $-0.127^{*}$ & $0.157^{*}$ & $-0.019^{*}$ & $0.027^{*}$ & $0.095^{*}$ \\
Words in triplets / 19 & $0.092^{*}$ & $-0.143^{*}$ & $0.092^{*}$ & $-0.056^{*}$ & $-0.013^{*}$ & $0.052^{*}$ \\
Two instruments / 17 & $0.135^{*}$ & $0.202^{*}$ & $-0.118^{*}$ & $0.417^{*}$ & $0.154^{*}$ & 0.478 \\
Saxophone plus noise / 19 & $0.147^{*}$ & $0.101^{*}$ & $-0.013^{*}$ & $0.203^{*}$ & $0.137^{*}$ & $0.133^{*}$ \\
Everyday sounds / 23 & -0.473 & -0.380 & $-0.247^{*}$ & -0.377 & -0.395 & $-0.310^{*}$
\end{tabular}

The subjects participated in six listening tests. The numbers of participants in each test are given in Table II. All of the tests were parts of other research (not yet published). Three consisted in the recognition of speech in babble noise. In one test, individual words out of the list of 21 spoken by one female speaker were presented at the background of white noise shaped in frequency according to the spectral envelope of the speaker's voice. This test is further denoted as "individual words". In another, triplets of words were presented against a background 
"babble" noise which was a fragment of a signal consisting of 5000 words spoken by the same female speaker and randomly scattered over a period of $10 \mathrm{~s}$. In this test, two sets of results were calculated: rate of recognition of individual words (further denoted as "words in triplets") and rate of recognition of entire triplets ("triplets"). The psychophysical method of constant stimuli was used in those tests, and the speech-to-noise ratio was varied in steps of $1.5 \mathrm{~dB}$.

In the other three tests non-speech stimuli were used: music excerpts and everyday sounds. In those tests, the task was to recognise a subtle effect introduced by a specific type of processing, consisting in the removal of any spectro-temporal overlap between sounds from different sources [9]. This effect is perceptually similar to the effects sometimes audible after digital coding of audio. In the first of these tests the removal of spectro-temporal overlap was applied to a mixture of a saxophone and a synthesizer sound (further denoted as "two instruments"), in the second to a mixture of a saxophone sound and specifically added white noise ("saxophone plus noise"), in the third to a mixture of everyday sounds: the rattling of dishes plus rippling of water ("everyday sounds"). In those three tests the "same-different" scheme was used within the "one alternative, forced choice" (1AFC) paradigm, where the stimuli were presented in pairs: original and processed. The task of the listeners was to decide whether the stimuli in a pair were the same or different.

\section{Results}

For each of the six types of listening tasks and for each of the six hearing acuity estimates a functional relationship was drawn: performance in the listening task measured by percent correct answers vs. hearing acuity estimate. For each of those relationships the correlation coefficient was calculated. The results are shown in Table II.

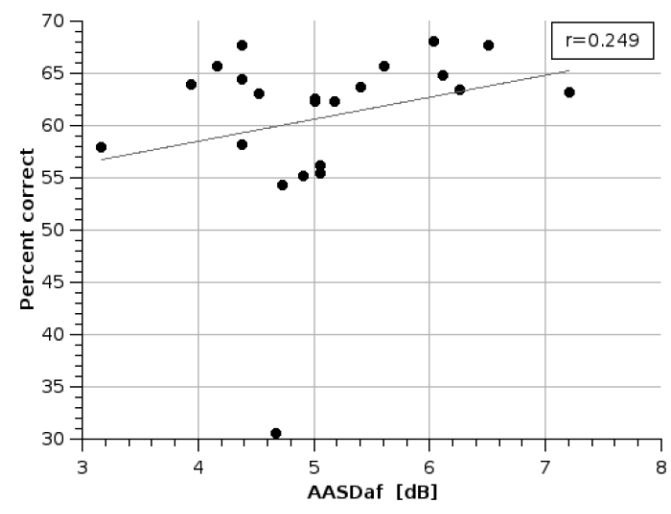

Fig. 1. The relationship between results of the test "individual words" and hearing acuity according to AASDaf parameter as described in Sect. 2 .

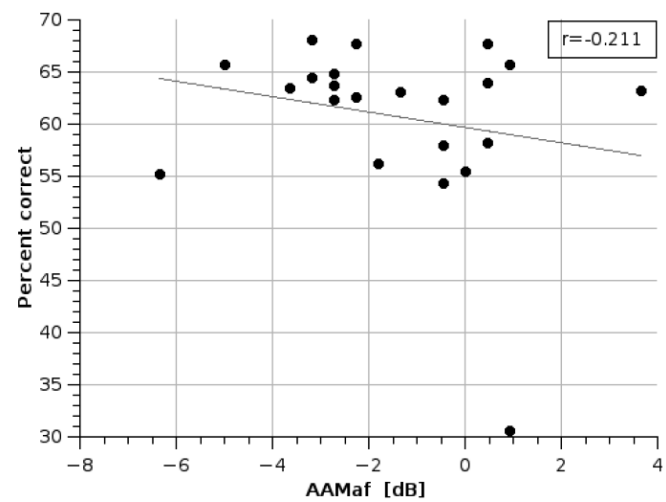

Fig. 2. As in Fig. 1, but for AAMaf parameter.

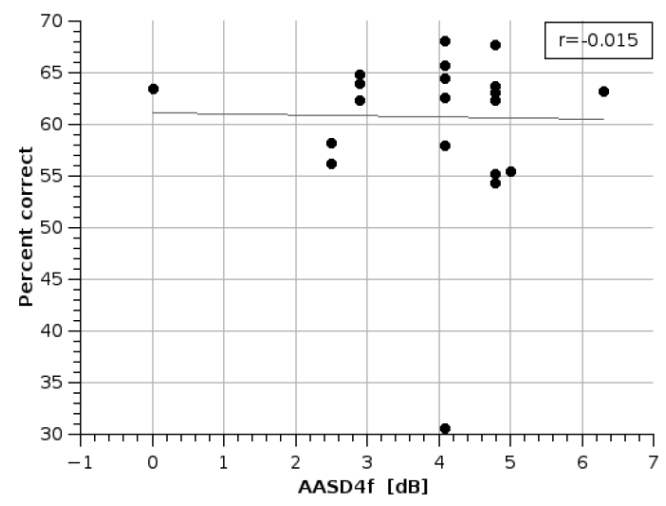

Fig. 3. As in Fig. 1, but for AASD4f parameter.

The results are presented graphically for all relationships based on "individual words" (Figs. 1-6). Some other relationships are presented in Figs. 6-12. The linear regression fits to data points and values of correlation are included.

Out of 36 correlations in Table II only four entries for "everyday sounds" are statistically significant at $p<0.05$, and one entry for "two instruments" (AMM4flo averaging).

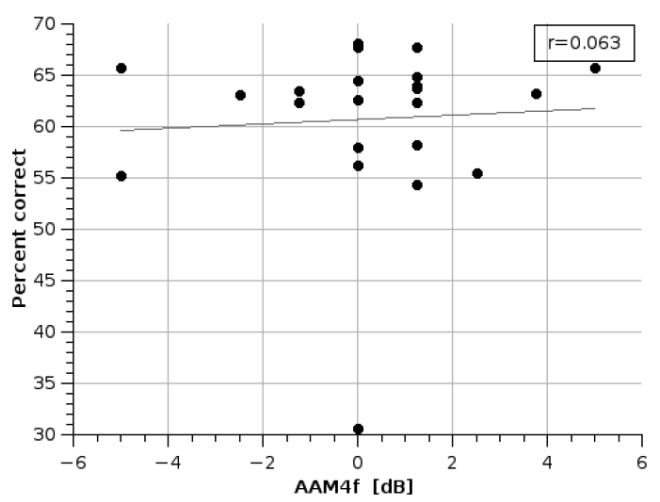

Fig. 4. As in Fig. 1, but for AAM4f parameter. 


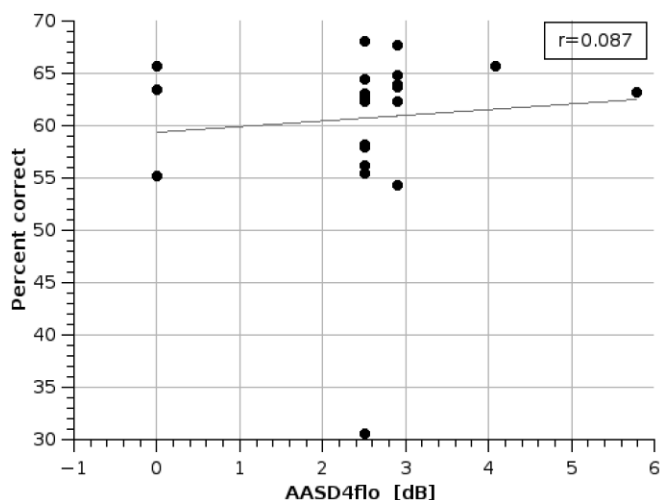

Fig. 5. As in Fig. 1, but for AASD4flo parameter.

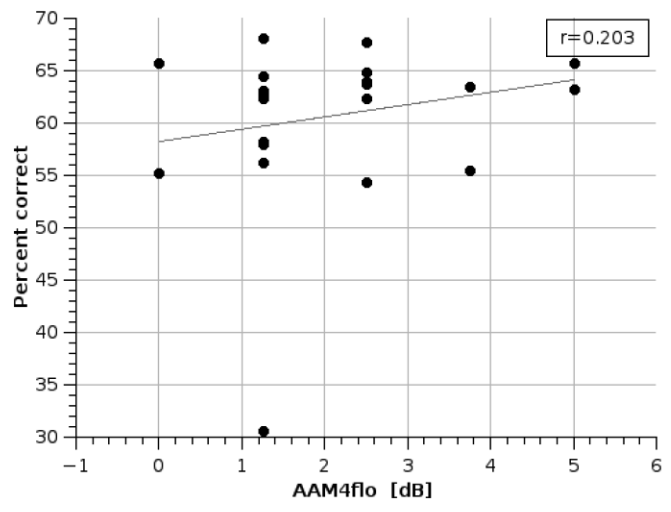

Fig. 6. As in Fig. 1, but for AAM4flo parameter.

\section{Discussion}

It can be observed that no correlation occurs in general. The only systematic but medium correlation occurs for "everyday sounds". This correlation is significant in 4 out of 6 cases and is uniformly negative for all six methods of averaging. Negative signs of correlations in functional relationships depicted in the figures are the

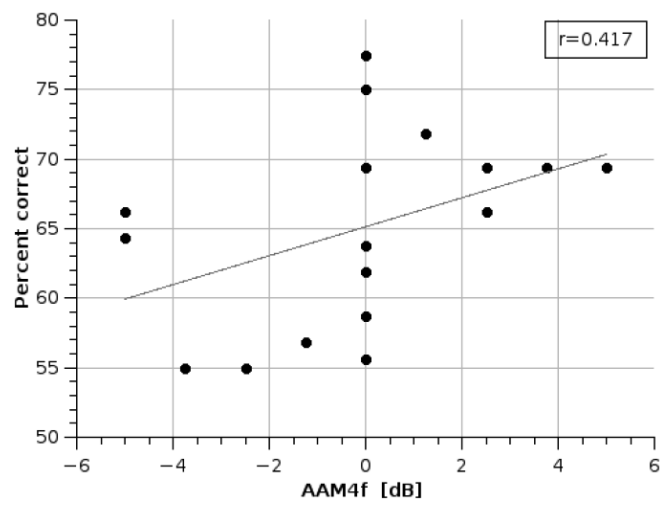

Fig. 7. The relationship between results of the test "two instruments" and hearing acuity according to AAM4f parameter.

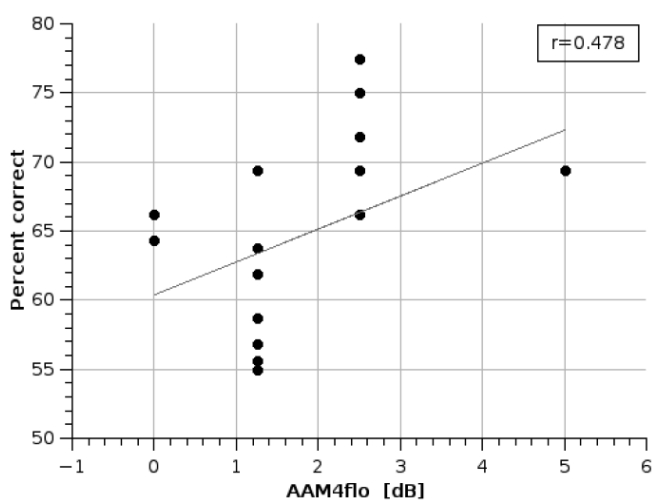

Fig. 8. As in Fig. 7, but for AAM4flo parameter.

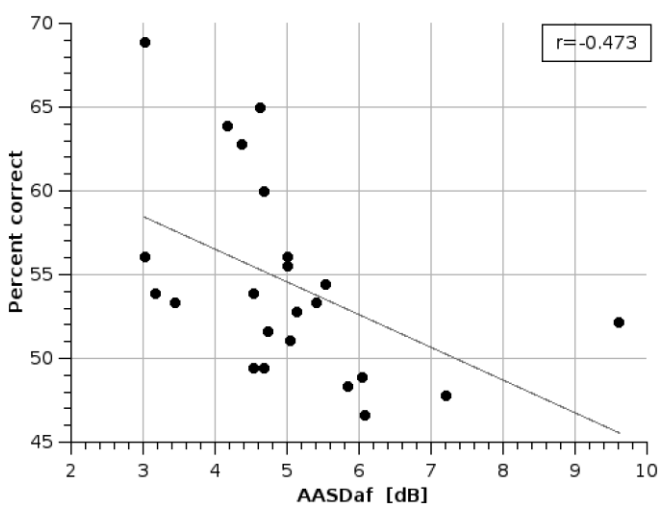

Fig. 9. The relationship between results of the test "everyday sounds" and hearing acuity according to AASDaf parameter.

result of the abscissa values increasing from the left to right. In fact this indicates that better parameters of hearing correspond to better results in listening tasks with everyday sounds. For all other types of stimuli there is no indication that better parameters of hearing help listeners perform better in listening tasks. When types of averaging of audiograms are compared, no ad-

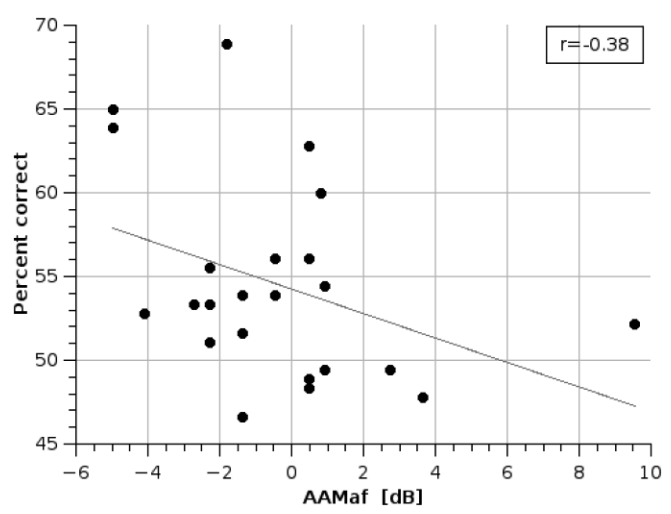

Fig. 10. As in Fig. 9, but for AAMaf parameter. 


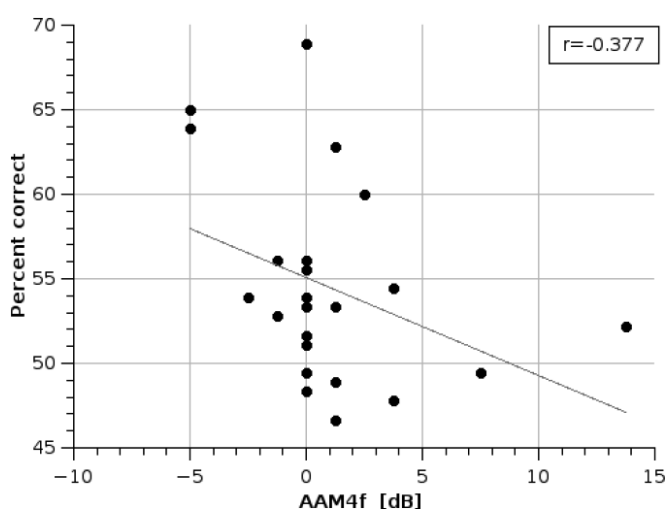

Fig. 11. As in Fig. 9, but for AAM4f parameter.

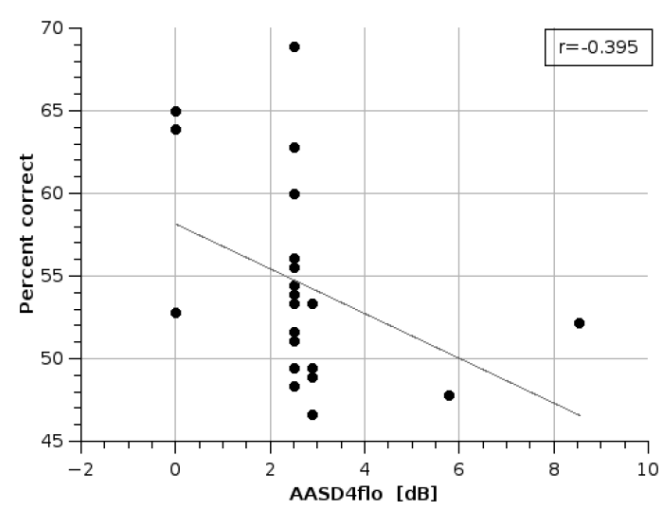

Fig. 12. As in Fig. 9, but for AASD4flo parameter.

vantage of any particular method can be noticed. Each of the methods yielded just one statistically significant correlation.

\section{Conclusions}

When audiograms of normally hearing subjects are evaluated with a single parameter, no significant relationship between hearing acuity and the performance in listening tests can be observed. No particular efficiency in revealing this relationship was observed when comparing six methods of averaging, three of them based on standard deviation i.e. measuring "flatness" of audiograms.

An interesting exception to this rule are everyday sounds, which are recognised more efficiently by listeners with lower average threshold level and more flat audiograms.

\section{Acknowledgments}

This work was supported by the National Centre for Research and Development, grant no. R02 0030 06/2009.

\section{References}

[1] H. Fletcher, J. Acoust. Soc. Am. 22, 1 (1950).

[2] A.N.S.I., Hearing impairment calculation worksheet www. Ini .wa.gov/Forms/pdf/252007a0.pdf, access 12.17.2011.

[3] R. Carhart, J. Acoust. Soc. Am. 17, 339 (1946).

[4] B.M. Siegenthaler, R. Strand, J. Acoust. Soc. Am. 36, 589 (1964).

[5] S. Bech, N. Zacharov, Perceptual Audio Evaluation: Theory, Method and Application, Wiley, Hoboken 2006.

[6] F.E. Toole, J. Audio Eng. Soc. 33, 2 (1985).

[7] S. Bech, J. Audio Eng. Soc. 40, 590 (1992).

[8] S. Shlien, G. Souloudre, in: 101 AES Conv., Audio Engineering Society, Los Angeles 1996, paper 4339.

[9] P. Kleczkowski, in: 119 AES Conv., Audio Engineering Society, New York 2005, paper 6552. 